\title{
Transcriptome Analysis of Salt Stress Response in Roots of Halophyte Zoysia macrostachya
}

\author{
Huaguang $\mathrm{Hu}^{1,2^{*}}$, Zhenming Zhang ${ }^{2}$ and Shengnan $\mathrm{Min}^{2}$ \\ ${ }^{1}$ Jiangsu Key Laboratory for Bioresources of Saline Soils, Yancheng Teachers University, Yancheng 224007, China \\ ${ }^{2}$ School of Marine and Bioengineering, Yancheng Teachers University, Yancheng 224007, China \\ *For correspondence: hhgjoy@163.com \\ Received 11 September 2020; Accepted 12 November 2020; Published 25 January 2021
}

\begin{abstract}
Zoysia macrostachya Franch. et Sav. is a halophyte with very strong tolerance to salinity, which can serve as an alternative turfgrass for landscaping in saline-alkali land and provide the salt-tolerance genes for turfgrass breeding. To further illustrate the salt-tolerance mechanisms in this species at molecular level, the roots transcriptome of $Z$. macrostachya was investigated under salt stress using the Illumina sequencing platform. Altogether 47,325 unigenes were assembled, among which, 32,542 $(68.76 \%)$ were annotated, and $87.61 \%$ clean reads were mapped to the unigenes. Specifically, 14,558 unigenes were shown to be the differentially expressed genes (DEGs) following exposure to $710 \mathrm{mM} \mathrm{NaCl}$ stress compared with control, including 7972 up-regulated and 6586 down-regulated DEGs. Among these DEGs, 24 were associated with the reactive oxygen species (ROS) scavenging system, 61 were found to be related to $\mathrm{K}^{+}$and $\mathrm{Na}^{+}$transportation, and 16 were related to the metabolism of osmotic adjustment substances. Additionally, 2327 DEGs that encoded the transcription factors (TFs) were also identified. The expression profiles for 10 DEGs examined through quantitative real-time PCR conformed to the individual alterations of transcript abundance verified through RNA-Seq. Taken together, results of transcriptome analysis in this study provided useful insights for salt-tolerance molecular mechanisms of Z. macrostachya. Furthermore, these DEGs under salt stress provided important clues for future salt-tolerance genes cloning of Z. macrostachya. (C) 2021 Friends Science Publishers
\end{abstract}

Keywords: Analysis; Roots; Salt stress; Transcriptome assemble; Zoysia macrostachya

\section{Introduction}

Soil salinization is not only a leading cause of the deteriorating ecological environment, but also a major abiotic factor affecting crop yield around the world (Zhu 2001). According to related statistics, there is $4.0 \times 10^{8} \mathrm{hm}^{2}$ saline-alkali land in the world, of which, an area of $3.6 \times 10^{7}$ $\mathrm{hm}^{2}$ is located in China (Zhang et al. 2007). The cultivation of new salt-tolerance plant varieties is an effective way to utilize the saline-alkali land. Meanwhile, investigating the salt-tolerance mechanisms in plant and identifying the salttolerance genes can lay a solid foundation for cultivating the new salt-tolerance plant varieties.

Research on the salt-tolerance mechanisms of plants had been carried out over the past many decades. Selective ion absorption and compartmentalization play key roles in maintaining ion homeostasis in cytoplasm. For instances, salt overly sensitive (SOS) and $\mathrm{Na}^{+} / \mathrm{H}^{+}$antiporter (NHX) can keep lower $\mathrm{Na}^{+}$content in cytoplasm (Zhu 2003), whereas the $\mathrm{K}^{+}$transporters with high affinity (HKTs) can improve $\mathrm{K}^{+}$and limit $\mathrm{Na}^{+}$transportation from root to leaf (Tang et al. 2015). Salt stress can induce ROS production, including hydrogen peroxide $\left(\mathrm{H}_{2} \mathrm{O}_{2}\right)$, superoxide radicals $\left(\cdot \mathrm{O}_{2}{ }^{-}\right)$and hydroxyl radicals $(\cdot \mathrm{OH})$, eventually causing oxidative damage to cytomembrane (Miller et al. 2008). For alleviating the ROS-induced peroxidation damage, two kinds of ROS scavenging systems have evolved in plant, including the non-enzymatic antioxidants and the enzymatic antioxidants. The non-enzymatic antioxidants include glutathione (GSH) and ascorbic acid (AsA) etc.; whereas the enzymatic antioxidants consist of peroxidase (POD), catalase (CAT) and superoxide dismutase (SOD) etc., and they play crucial roles in altering the ROS homeostasis (Deinlein et al. 2014). Salt stress leads to imbalanced osmotic regulation; as a result, some osmolytes, such as free proline, sugar and betaine, are synthesized in cells to regulate the osmotic balance in plants (Ingram and Bartels 1996; Ashraf and Foolad 2007). In addition, the expression of salt-tolerance genes in plants are regulated by transcription factors (TFs), and many of which, including DREB, MYB, AP2/ERF and NAC families etc, exert vital parts in plant tolerance to salt stress (Deinlein et al. 2014).

Halophyte is a plant that grows regularly and completes the life cycles under the single salt concentration of $>70 \mathrm{mmol} \cdot \mathrm{L}^{-1}$ (Flowers and Colmer 2008). $Z$. macrostachya, the perennial warm-season turfgrass native 
to China, Japan as well as Korean Peninsula, and mainly grows in the coastal wetlands of Shandong, Jiangsu and Zhejiang provinces in China. Z. macrostachya can rapidly spread through rhizomes and stolons to form the dense turf with the deep root system, which can thereby be used as the soil-conserving, dike-protecting and sand-fixing turf. Moreover, Z. macrostachya is an euhalophyte, which exhibits tolerance to salinity and may be potentially used in landscaping of saline-alkali land. According to our previous research, Z. macrostachya tolerated $355 \mathrm{mM} \mathrm{NaCl}$ stress (Hu and Zhang 2010) and further research found that the roots of Z. macrostachya limited $\mathrm{Na}^{+}$absorption while improved $\mathrm{K}^{+}$absorption and transportation from roots to leaves, accumulated free proline and soluble sugars and enhanced the POD activity under salt stress, and all of these improved the salt-tolerance of $Z$. macrostachya ( $\mathrm{Hu}$ and Zhang 2009, 2010; Hu et al. 2016). Nonetheless, the above studies are limited to physiological indexes, and the molecular mechanisms of salt-tolerance in this species remain unclear so far.

RNA-seq has emerged as a powerful tool to analyze genes expressional changes, which reflects the molecular mechanisms of plant response to salt stress, and has been utilized for investigating the molecular mechanisms of salttolerance in many halophytes, such as Iris lactea var. Chinensis (Gu et al. 2018), Prunellae Spica (Liu et al. 2020) and Rhizophora mucronata (Meera and Augustine 2020). To date, changes in global genes expression of $Z$. macrostachya under salt stress are still unknown, which limits the understanding towards the molecular mechanisms of salt-tolerance in this species. In this study, the Illumina HiSeq XTen sequencing platform was used to generate a roots reference transcriptome dataset and to explore DEGs with aims to improve our comprehensive understanding of the mechanisms of salt-tolerance at transcriptome level and to identify the DEGs involved in the salt-tolerance of $Z$. macrostachya.

\section{Materials and Methods}

\section{Plant material, salt stress and RNA extraction}

Zoysia macrostachya samples were collected from coastal wetland located $45.0 \mathrm{~km}$ east of Yancheng of Jiangsu province, China. Samples were brought back to the laboratory and planted in six PVC tubes $(35 \mathrm{~cm}$ in length and $15 \mathrm{~cm}$ in diameter) filled with river sand, respectively. The plants were grown in a growth chamber with a $12 \mathrm{~h}$ light $/ 12 \mathrm{~h}$ dark cycle, $30 / 20^{\circ} \mathrm{C}$ day/night temperature, 800 $\mu \mathrm{mol} \mathrm{m} \mathrm{m}^{-2} \cdot \mathrm{s}^{-1}$ light intensity and a relative humidity of $80 \%$. The plants were watered at intervals of three days, and irrigated weekly with $200 \mathrm{~mL} \mathrm{1/2}$ Hoagland's nutrient solution during growth. After $30 \mathrm{~d}$ of growth, the plants were pulled out from the tubes and river sand were washed by water. Then, the roots of Z. macrostachya were soaked in $710 \mathrm{mM} \mathrm{NaCl}$ solution (treatment) and distilled water (control), respectively. Three biological replications were set for each treatment, three treatments were marked as treatment $_{1}$, treatment $_{2}$, treatment $_{3}$; and three controls were marked as control 1 , $\operatorname{control}_{2}$, $\operatorname{control}_{3}$. After $8 \mathrm{~h}, 3 \mathrm{~cm}$ root tip were harvested and stored in liquid nitrogen for extracting RNA. Total RNA was extracted from three treatment and three control samples, respectively, using a mirVana miRNA Isolation Kit (Ambion) in accordance with the manufacturer's protocol.

\section{Preparation and sequencing of cDNA library}

The integrity of RNA was assessed by the Agilent 2100 Bioanalyzer (Agilent Technologies, Santa Clara, CA, USA). Afterwards, all samples with the RNA Integrity Number (RIN) of $\geq 7$ were used for constructing cDNA libraries. Six cDNA libraries were constructed by the TruSeq Stranded mRNA LTSample Prep Kit (Illumina, San Diego, CA, USA) in accordance with the manufacturer's protocol. The above libraries were sequenced using the Illumina HiSeq XTen system to generate the $125 / 150 \mathrm{bp}$ reads with paired-end.

\section{Quality control and de novo assembly}

Original data (raw reads) were processed by Trimmomatic (Bolger et al. 2014). Reads that contained ploy-N and were of low quality were eliminated to obtain clean reads. Later, the adaptor and sequences of low quality were removed, clean reads were assembled into expression sequence tag clusters (contigs), then de novo assembled into transcript through Trinity (version: trinityrnaseq_r20131110) according to the paired-end approach (Grabherr et al. 2011). Subsequently, transcript with the greatest length was selected as the unigene based on the sequence length and similarity.

\section{Function annotations}

Unigene functions were annotated through aligning them with SwissProt protein, NCBI non-redundant protein (NR), Clusters of orthologous groups for eukaryotic complete genomes (KOG) and Pfam databases using Blastx (Altschul et al. 1990), with the cut-off E-value of $10^{-5}$. Typically, those proteins with the greatest hits to the above unigenes were utilized for assigning the functional annotations. According to SwissProt annotations, the gene ontology (GO) analysis was performed based on mapping relation between SwissProt and GO terms. All unigenes were mapped to the Kyoto Encyclopedia of Genes and Genomes (KEGG) database for annotating the underlying metabolic pathways (Kanehisa et al. 2008).

\section{Unigenes quantification, analysis of DEGs, cluster analysis, GO and KEGG enrichment analyses}

FPKM value (Trapnell et al. 2010), together with the read counts for every unigene, were computed through eXpress 
(Roberts and Lior 2013) and bowtie2 (Langmead and Salzberg 2012). Afterwards, DEGs were identified through nbinom Test and DESeq (Anders and Huber 2012) functions estimate Size Factors. Unigenes with P-value of $\leq 0.05$ and $\log _{2}$ foldChange of $\geq 1$ were selected as thresholds of significant DEGs. Later, DEGs were performed hierarchical cluster analysis for exploring the expression profiles of transcripts. Then, the DEGs were carried out for GO enrichment and KEGG pathway analyses using the R software based on the hypergeometric distribution.

\section{Annotation of TFs}

The Hidden Markov Model (HMM) motif sequences of TFs were obtained based on Plant Transcription Factor Database (TFDB), which contained $58 \mathrm{TF}$ families of green plant (Jin et al. 2014). Specifically, one unigene with $\geq 90 \%$ sequence homology was annotated as the putative TF (E-value of $\leq 1 \mathrm{E}^{-10}$ ). Thereafter, DEGs, together with the candidate TFs in reference transcriptome, were clustered according to the TF families.

\section{Quantitative real-time PCR (qRT-PCR)}

For further validating the results of transcriptomic analyses, 10 DEGs including 1 POD-related, 2 SOD-related, 2 KEArelated, 2 KUP-related, 1 KOC-related, 1 Proline-related and 1 Betaine-related DEGs were selected for qRT-PCR analysis. Z. macrostachya were in exposure of $710 \mathrm{mM}$ $\mathrm{NaCl}$ solution and distilled water for $8 \mathrm{~h}$, respectively, then the root RNA were extracted according to the method mentioned above. Reverse transcription reactions were performed using SuperScript III reverse transcriptase (Invitrogen, Grand Island, NY, USA) following the manufacturer's instructions. Primers (Table 1) for qRT-PCR were designed using the Premier v5.0 software (Premier Biosoft, Palo Alto, CA, USA) with $\beta$-actin genes as the internal controls. The qRT-PCR was carried out by the Twocolor Real-time PCR Detection System (Bio-Rad, USA) following amplification protocol: $3 \mathrm{~min}$ at $95^{\circ} \mathrm{C}$, and then $3 \mathrm{~s}$ at $95^{\circ} \mathrm{C}$ and $30 \mathrm{~s}$ at $60^{\circ} \mathrm{C}$ for 45 cycles. All reactions were performed in triplicate, the relative expression levels of the selected unigenes normalized to $\beta$-actin was calculated using the $2^{-} \Delta \Delta^{\mathrm{Ct}}$ method (Livak and Schmittgen 2001).

\section{Results}

\section{Sequencing results and assembly}

A mean of 49.49 million raw reads were produced from controls, whereas 49.44 million raw reads were produced from $710 \mathrm{mM} \mathrm{NaCl}$ treatments (Table 2). Over $93.99 \%$ of all raw reads possessed the Phred-like quality score of Q30 level (error probability $=1 \%$ ). An average of 48.23 million (controls) and 48.19 million (treatments) clean reads were obtained, and the valid base ratio and GC content were above

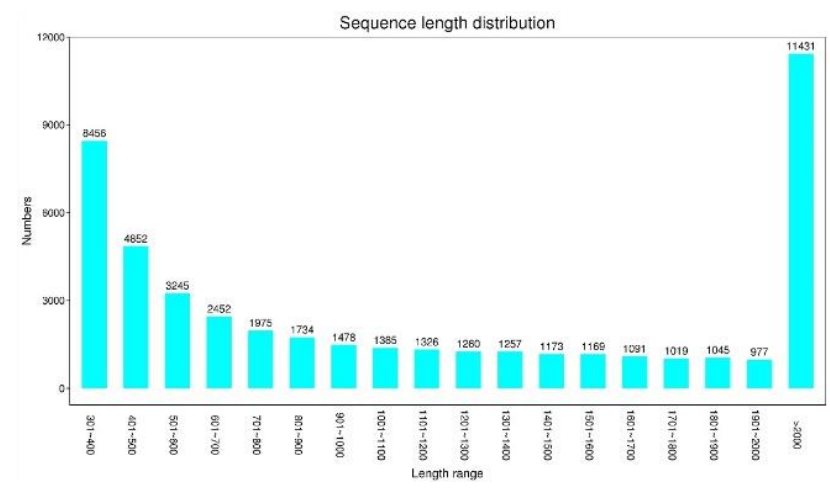

Fig. 1: Unigene length distribution

95.28 and $51.05 \%$, respectively, indicating high quality of sequencing and cDNA library establishment. A total of 4,7325 unigenes with mean length of $1397 \mathrm{bp}$ and the N50 of 2169 bp were obtained. As shown in Fig. 1, 24,192 unigenes had the length of 301-1000 bp, 10,317 had the length of $1001-2000 \mathrm{bp}$, and 11,431 had the length of $>2000 \mathrm{bp}$.

Clean reads of each sample were mapped to $Z$. macrostachya unigenes to confirm the quality of sequencing for those six samples (Table 3). As observed, the average total mapped reads proportion was $87.61 \%$ (range, 86.98 $88.44 \%$ ), and the multi-position matched reads accounted for approximately $21.65 \%$, and $64.89-67.06 \%$ reads were uniquely matched unigenes in all the six samples. An average of $81.43 \%$ reads was mapped in pairs.

\section{Functional annotations of unigenes}

Unigenes were annotated by aligning to the publicly accessible databases (Table 4). Estimated number of 32,033 $(67.69 \%)$ unigenes were aligned in the NR database, 24,155 $(51.04 \%)$ in the Swissprot database, 11,601 $(24.51 \%)$ in the KEGG database, 17,906 (26.78\%) in the KOG database, $22,309(47.14 \%)$ in the GO database, and $51(0.11 \%)$ in the Pfam database. Altogether 32,542 unigenes (68.76\%) were annotated against at least one of the following databases, including NR, SwissProt, KEGG, Pfam, GO and KOG.

According to the homology of sequences, 22,309 unigenes were clustered to 3 major GO classifications, including biological process, cellular component and molecular function (Fig. 2). These unigenes were subdivided into $57 \mathrm{GO}$ terms. Among biological process classifications, the term of "biological regulation", "cellular process", "metabolic process", "regulation of biological process" and "responses to stimulus" were the dominant clusters, whereas only a few unigenes belonged to the "biological adhesion", "cell killing", "locomotion" and "rhythmic process" term. With regard to the cellular component classification, the term of "cell", "cell part" and "organelle" had the bigger unigenes proportions. As to the molecular function, most unigenes were classified into the "binding", "catalytic activity" and "transporter activity" 
Hu et al. / Intl J Agric Biol, Vol 25, No 3, 2021

Table 1: The qRT-PCR primers for detecting the accuracy of transcriptome data

\begin{tabular}{lllll}
\hline Unigene number & Unigene name & Annotated as & Forward primers $\left(5^{\prime}-3{ }^{\prime}\right)$ & Reverse primers $\left(5^{\prime}-3^{\prime}\right)$ \\
\hline 1 & TRINITY_DN11437_c0_g1_i1_2 & POD & GCAAGTCAAGCACCTCAACCT & TGATTGGAATGGTCTGCTGGGA \\
2 & TRINITY_DN10853_c0_g1_i2_1 & SOD & GACTGAATCTCTACGCCTGT & GACGACGTATGGCACCAGAG \\
3 & TRINITY_DN19735_c0_g1_i6_2 & SOD & AGCTGCTCCATTGCCATTCCT & CCGAACCTCCTGTAAGTCAACC \\
4 & TRINITY_DN19255_c0_g2_i4_1 & KEA & TAGTAAGGGAGAATCTTTGCAAG & ATGGCAGATCCGTGACAAGTC \\
5 & TRINITY_DN22016_c0_g1_i24_2 & KEA & TCACCGCCATCCCAGTCATC & CCCAAATACACGCACTCCTG \\
6 & TRINITY_DN16474_c0_g3_i1_2 & KUP & CTTGTCGACCAACATTCACACC & CGAGTAAGCAACGGTCCA \\
7 & TRINITY_DN16569_c0_g2_i2_1 & KUP & ACTGGACGAGGAGCAACACT & TCTCACTCACTCCTCCAGAGC \\
8 & TRINITY_DN16911_c0_g2_i5_2 & KOC & TCCCAAACCAGCTTCACCGATT & CACCAGAGTATGCCTCGACAT \\
9 & TRINITY_DN20583_c0_g1_i5_1 & Free proline & TTACAACGGTTCGCAACTGT & TGCGTGGACTACTCAGAGACC \\
10 & TRINITY_DN15030_c0_g1_i1_1 & Glycine betaine & TCTCCTCATCCTCACCTCAT & ATTGCCAGTTCCTAGTGTTCC \\
\hline
\end{tabular}

Table 2: Summary of the sequence analysis

\begin{tabular}{|c|c|c|c|c|c|c|c|}
\hline Sample & Raw reads & Raw bases & Clean reads & Clean bases & Valid bases & Q30 & GC \\
\hline Control $_{1}$ & 49536428 & 7430464200 & 48215536 & 7090647459 & $95.43 \%$ & $94.42 \%$ & $51.16 \%$ \\
\hline $\mathrm{Control}_{2}$ & 49533550 & 7430032500 & 48398632 & 7115121942 & $95.76 \%$ & $94.68 \%$ & $51.84 \%$ \\
\hline $\mathrm{Control}_{3}$ & 49424632 & 7413694800 & 48083930 & 7063618562 & $95.28 \%$ & $93.99 \%$ & $51.40 \%$ \\
\hline Treatment $_{2}$ & 49108932 & 7366339800 & 47923128 & 7058090438 & $95.82 \%$ & $94.58 \%$ & $51.05 \%$ \\
\hline Treatment $_{3}$ & 49536672 & 7430500800 & 48291630 & 7112106699 & $95.72 \%$ & $94.38 \%$ & $51.26 \%$ \\
\hline
\end{tabular}

Table 3: Mapping of clean reads to unigenes

\begin{tabular}{|c|c|c|c|c|c|c|}
\hline Terms $\backslash$ Samples & Control $_{1}$ & $\mathrm{Control}_{2}$ & $\mathrm{Control}_{3}$ & Treatment $_{1}$ & Treatment $_{2}$ & Treatment $_{3}$ \\
\hline Total reads & $\begin{array}{l}48215536 \\
(100.00 \%)\end{array}$ & $\begin{array}{l}48398632 \\
(100.00 \%)\end{array}$ & $\begin{array}{l}48083930 \\
(100.00 \%)\end{array}$ & $\begin{array}{l}48368100 \\
(100.00 \%)\end{array}$ & $\begin{array}{l}47923128 \\
(100.00 \%)\end{array}$ & $\begin{array}{l}48291630 \\
(100.00 \%)\end{array}$ \\
\hline Total mapped reads & $\begin{array}{l}42448603 \\
(88.04 \%)\end{array}$ & $\begin{array}{l}42802853 \\
(88.44 \%)\end{array}$ & $\begin{array}{l}42159835 \\
(87.68 \%)\end{array}$ & $\begin{array}{l}42069655 \\
(86.98 \%)\end{array}$ & $\begin{array}{l}41718189 \\
(87.05 \%)\end{array}$ & $\begin{array}{l}42224960 \\
(87.44 \%)\end{array}$ \\
\hline Multiple mapped & $\begin{array}{l}10306193 \\
(21.38 \%)\end{array}$ & $\begin{array}{l}10344313 \\
(21.37 \%)\end{array}$ & $\begin{array}{l}10207699 \\
(21.23 \%)\end{array}$ & $\begin{array}{l}10490280 \\
(21.69 \%)\end{array}$ & $\begin{array}{l}10622363 \\
(22.17 \%)\end{array}$ & $\begin{array}{l}10640511 \\
(22.03 \%)\end{array}$ \\
\hline Uniquely mapped & $\begin{array}{l}32142410 \\
(66.66 \%)\end{array}$ & $\begin{array}{l}32458540 \\
(67.06 \%)\end{array}$ & $\begin{array}{l}31952136 \\
(66.45 \%)\end{array}$ & $\begin{array}{l}31579375 \\
(65.29 \%)\end{array}$ & $\begin{array}{l}31095826 \\
(64.89 \%)\end{array}$ & $\begin{array}{l}31584449 \\
(65.40 \%)\end{array}$ \\
\hline Reads mapped in proper pairs & $\begin{array}{l}39451806 \\
(81.82 \%)\end{array}$ & $\begin{array}{l}39954228 \\
(82.55 \%)\end{array}$ & $\begin{array}{l}39326280 \\
(81.79 \%)\end{array}$ & $\begin{array}{l}38891334 \\
(80.41 \%)\end{array}$ & $\begin{array}{l}38763888 \\
(80.89 \%)\end{array}$ & $\begin{array}{l}39160458 \\
(81.09 \%)\end{array}$ \\
\hline
\end{tabular}

Table 4: Blast analysis of non-redundant unigenes against public databases

\begin{tabular}{llll}
\hline Databases for annotation & Unigene numbers & Percentages & $300 \leq$ Length<1000nt \\
\hline Annotated in NR & 32033 & $67.69 \%$ & 10665 \\
Annotated in Swissprot & 24155 & $51.04 \%$ & 6615 \\
Annotated in KEGG & 11601 & $24.51 \%$ & 3571 \\
Annotated in KOG & 17906 & $37.84 \%$ & 4774 \\
Annotated in GO & 22309 & $47.14 \%$ & 6231 \\
Annotated in Pfam & 51 & $0.11 \%$ & 41 \\
Annotated in at least one database & 32542 & $68.76 \%$ & 21547 \\
\hline
\end{tabular}

term. Interestingly, 225 unigenes were categorized into the "antioxidant activity" term, while 894 as the "transporter activity" term.

The KOG database was searched to identify unigenes to predict and classify their functions. Based on the sequence homology, 17,906 sequences showed a KOG classification (Fig. 3). Among the $25 \mathrm{KOG}$ clusters, the cluster of "general function prediction only" (3674, 20.52\%) was the largest group, followed by the "posttranslational modification, protein turnover, chaperones" (2180, 12.17\%), the "signal transduction mechanisms" $(1821,10.17 \%)$ and the "translation, ribosomal structure and biogenesis" (1266, $7.07 \%)$. By contrast, the clusters of the "cell motility" (3, $0.17 \%$ ), the "nuclear structure" $(51,0.28 \%)$ and the "extracellular structures" (52, 0.29\%) were smaller groups.
A total of 11,601 annotated unigenes were mapped to the reference canonical pathways in the KEGG database (Fig. 4). The most represented pathways were "metabolism" (5711 unigenes), "genetic information processing" (2822 unigenes), "cellular processes" (1430 unigenes) and "environmental information processing" (1298 unigenes). In addition, "translation" (1335 unigenes), "signal transduction" (1216 unigenes) and "carbohydrate metabolism" (1049 unigenes) were the pathways most closely related to the mapped unigenes, which suggested the presence of active pathways in Z. macrostachya.

\section{Recognition and annotation of DEGs}

A total of 14,558 DEGs were recognized, among which, 7972 were up-regulated and 6586 were down-regulated 


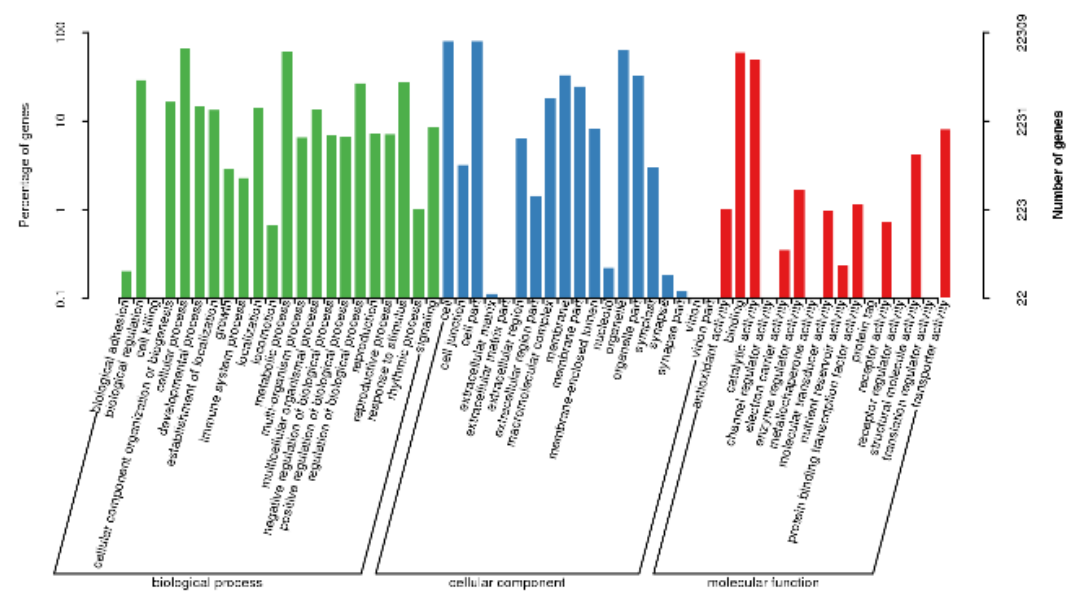

Fig. 2: GO categorization of unigenes

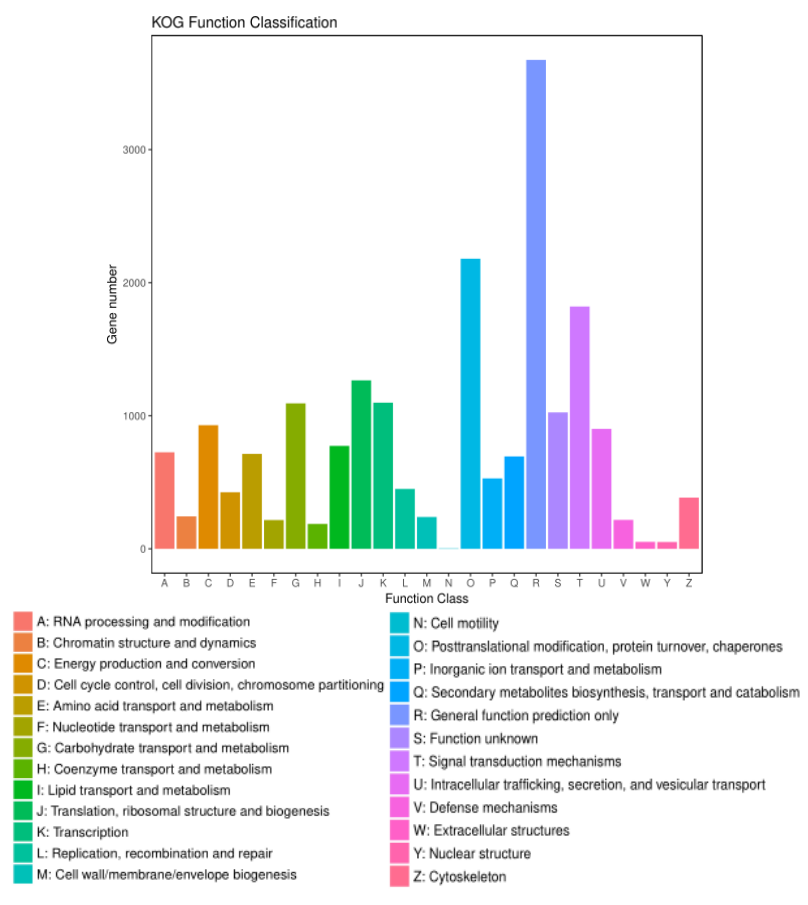

Fig. 3: KOG classification of unigenes

(Fig. 5). According to our findings, 7424 DEGs were enriched into 64 GO terms. With regard to biological process, the term of "cellular process", "metabolic process", "biological regulation", "regulation of biological process" and "response to stimulus" had the greater unigenes proportions. As to cellular component, the "membrane", the "cell part" and the "cell" term were the dominant clusters. The "binding", "catalytic activity" and "transporter activity" term contained more unigenes in the molecular function category (Fig. 6).

In addition, DEGs were performed for KEGG analysis. The results suggested that, 2093 DEGs were assigned with the KEGG ID, which were classified into 197

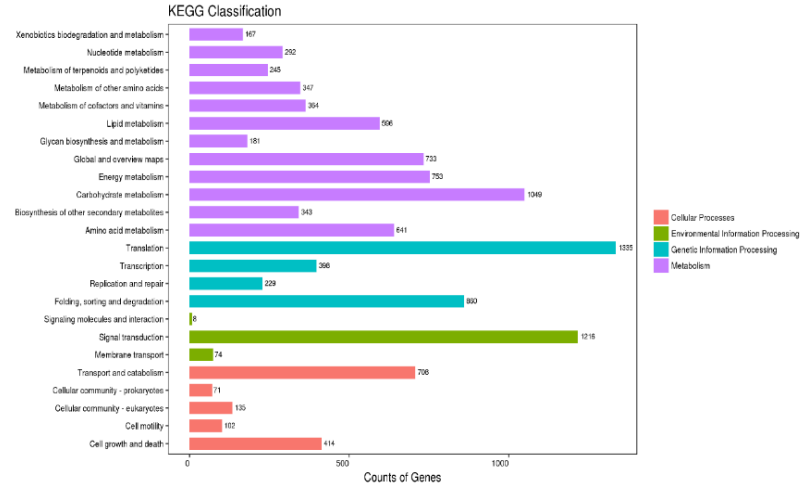

Fig. 4: KEGG categorization of unigenes

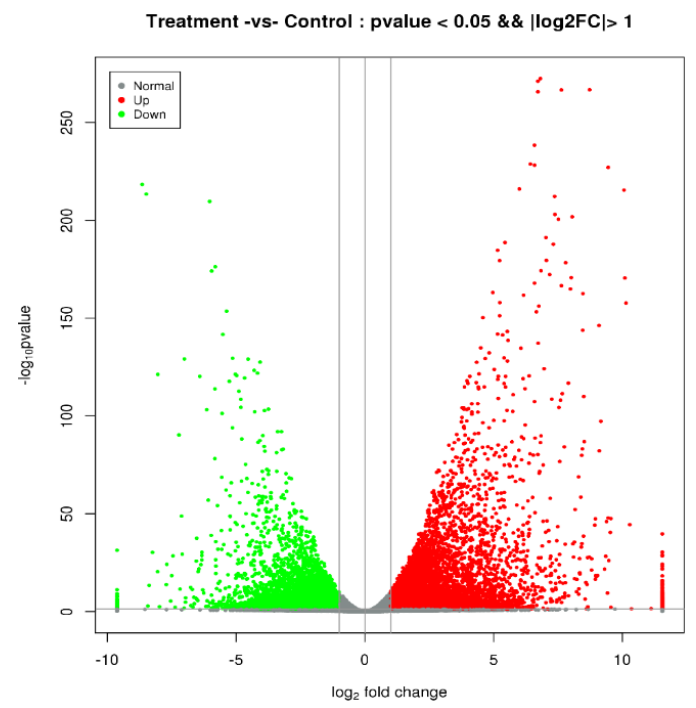

Fig. 5: Volcano plots of DEGs

pathways (Fig. 7). Typically, the most representative pathways were the "environmental information processing-signal transduction" (370 unigenes), the 


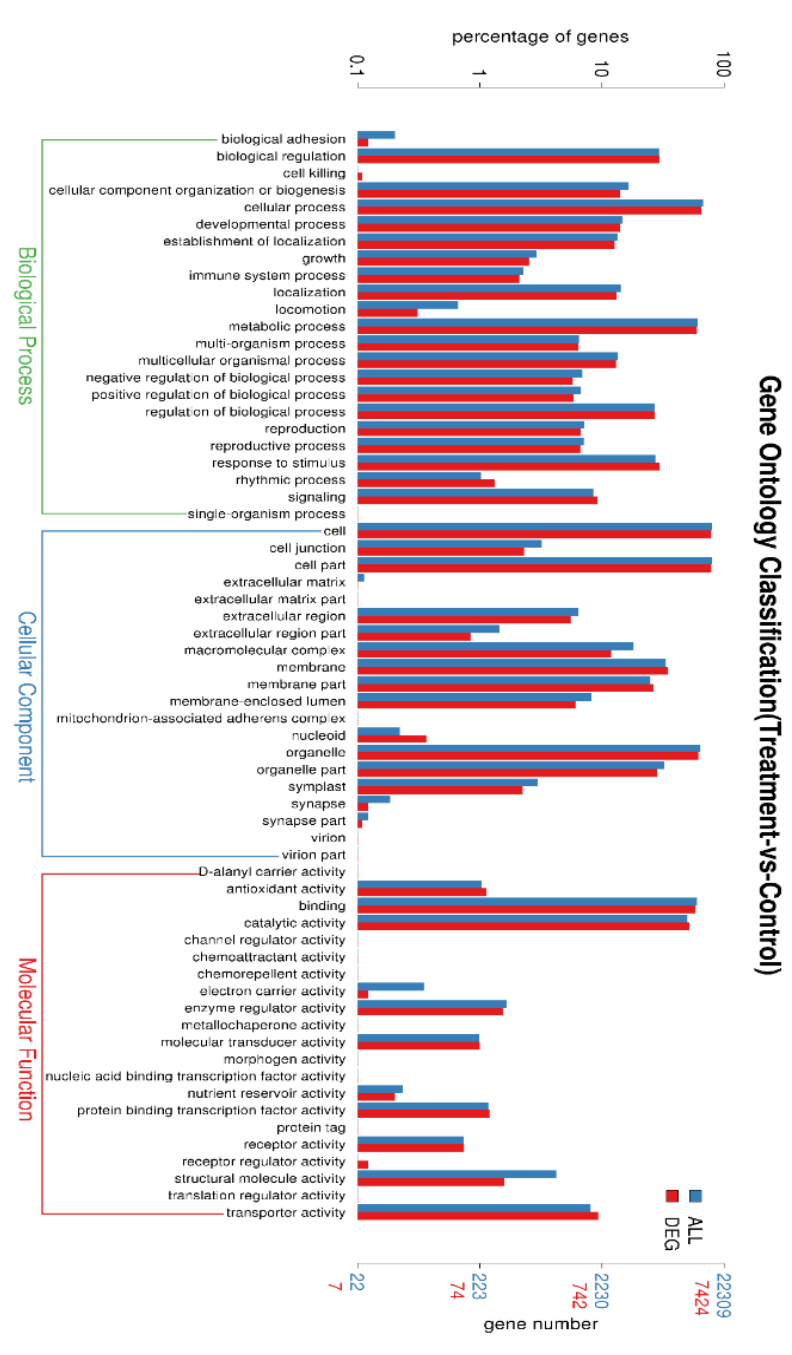

Fig. 6: GO categorization of DEGs in Z. macrostachya. under salt stress

"metabolism-amino acid metabolism" (248 unigenes), the "genetic information processing-translation" (224 unigenes), the "metabolism-global and overview maps" (223 unigenes) and the "metabolism-lipid metabolism" (219 unigenes).

\section{DEGs associated with ROS scavenging}

A total of 24 DEGs were identified to encode ROS scavenging-associated enzymes (Table 5). Among which, 12 encoded PODs and constituted the largest group. The expression of TRINITY_DN11379_c0_g1_i1_2, TRINITY_DN11437_c0_g1_i1_2 and TRINITY_DN12963_c0_g1_i2_2 increased under salt stress, with high transcript levels. Four genes encoded SODs, the expression of one gene increased, while that of the other three genes decreased. Two genes encoded CATs, among which, TRINITY_DN21281_c0_g4_i5_2 showed higher transcript abundance, but TRINITY_DN21281_c0_g1_i8_2 was down-regulated

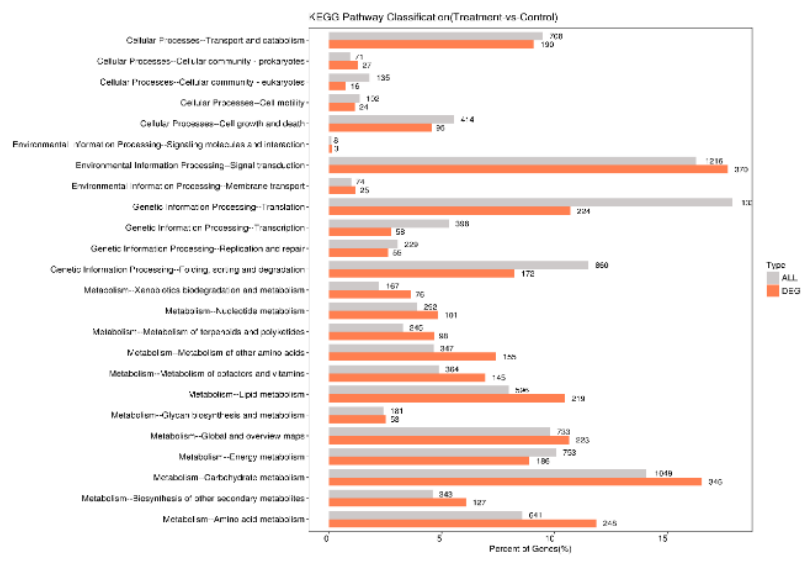

Fig. 7: KEGG categorization of DEGs in Z. macrostachya under salt stress

under salt stress. Additionally, five genes were found to encode Ascorbate peroxidase (APX) and one gene encoded glutathione peroxidase (GPX). The encoded APX gene TRINITY_DN19584_c0_g2_i1_2 was up-regulated by nearly 26 times (FC 26.80), with the highest transcript levels.

\section{DEGs involved in ion transportation}

Fifty-two DEGs were identified to be related to the regulation of $\mathrm{K}^{+}$transportation (Table 6), which accounted for the greatest proportion of identified genes. 37 out of them were up-regulated, while 15 were down-regulated under salt stress. Eleven of them were involved in $\mathrm{K}^{+}$efflux antiporter (KEA), twenty were related to $\mathrm{K}^{+}$transmembrane transporter (KUP), three were associated with $\mathrm{K}^{+}$channel $(\mathrm{AKT})$, nine were involved in the outward rectifying $\mathrm{K}^{+}$ channel (KOC) and nine were related to cyclic nucleotidegated channel (CNGC). Three genes were identified to encode the plasma membrane P-ATPases (PM- $\mathrm{H}^{+}$ATPases), while five encoded the vacuolar V-ATPases (V$\mathrm{H}^{+}$-ATPases), and these unigenes were up-regulated. Two unigenes that encoded $\mathrm{Na}^{+} / \mathrm{H}^{+}$exchanger (NHX) were also identified, but their expressions levels were down-regulated.

\section{DEGs associated with osmotic adjustment}

In this study, 12 DEGs were identified to involve in free proline metabolism, eight out of them were up-regulated, while four were down-regulated under salt stress (Table 7). Four DEGs were identified to involve in Glycine betaine metabolism, 3 out of them were up-regulated, only TRINITY_DN15030_c0_g1_i1_1 was down-regulated under salt stress (Table 7).

\section{DEGs related to TFs}

A total of 2,327 DEGs were annotated to the TFs database, which belonged to 57 families (Fig. 8). 604 out of them were annotated to the BHLH family, which accounted for 
Transcriptome of Zoysia macrostachya Roots under Salt Stress / Intl J Agric Biol, Vol 25, No 3, 2021

Table 5: DEGs related to the ROS scavenging system

\begin{tabular}{|c|c|c|c|c|c|}
\hline Gene ID & $\log _{2} \mathrm{FC}$ & P-value & Gene ID & $\log _{2} \mathrm{FC}$ & P-value \\
\hline POD & & & SOD & & \\
\hline TRINITY_DN10724_c0_g1_i2_1 & -1.61 & $1.46 \mathrm{E}-02$ & TRINITY_DN10853_c0_g1_i2_1 & -2.67 & $1.13 \mathrm{E}-11$ \\
\hline TRINITY_DN11379_c0_g1_i1_2 & 4.18 & $2.69 \mathrm{E}-11$ & TRINITY_DN19735_c0_g1_i6_2 & 1.97 & 3.98E-04 \\
\hline TRINITY_DN11437_c0_g1_i1_2 & 5.43 & $9.90 \mathrm{E}-03$ & TRINITY_DN699_c0_g1_i1_2 & -1.13 & $5.85 \mathrm{E}-06$ \\
\hline TRINITY_DN11915_c0_g1_i1_1 & -2.11 & $1.28 \mathrm{E}-25$ & TRINITY_DN7158_c0_g1_i2_1 & -2.22 & $1.73 \mathrm{E}-11$ \\
\hline TRINITY_DN12563_c0_g1_i1_1 & -3.11 & 2.07E-16 & APX & & \\
\hline TRINITY_DN12830_c0_g1_i3_1 & 1.20 & 4.15E-07 & TRINITY_DN18445_c0_g1_i11_1 & -4.10 & $1.56 \mathrm{E}-08$ \\
\hline TRINITY_DN12963_c0_g1_i2_2 & 4.89 & 5.57E-14 & TRINITY_DN18597_c0_g2_i1_2 & 1.56 & $1.30 \mathrm{E}-02$ \\
\hline TRINITY_DN13326_c0_g1_i1_2 & 1.42 & $2.82 \mathrm{E}-02$ & TRINITY_DN19584_c0_g1_i3_2 & 1.99 & $1.39 \mathrm{E}-29$ \\
\hline TRINITY_DN13354_c0_g1_i1_1 & -3.66 & $5.97 \mathrm{E}-12$ & TRINITY_DN19584_c0_g2_i1_2 & 4.74 & $1.61 \mathrm{E}-03$ \\
\hline TRINITY_DN14879_c0_g3_i1_1 & -2.59 & $1.10 \mathrm{E}-15$ & TRINITY_DN9766_c0_g1_i2_2 & -2.91 & 7.99E-46 \\
\hline TRINITY_DN14924_c0_g1_i1_1 & -2.67 & $2.84 \mathrm{E}-02$ & GPX & & \\
\hline TRINITY_DN15006_c1_g1_i1_1 & 2.78 & 7.16E-11 & TRINITY_DN13470_c0_g1_i1_1 & 1.21 & $9.32 \mathrm{E}-05$ \\
\hline \multicolumn{6}{|l|}{ CAT } \\
\hline TRINITY_DN21281_c0_g1_i8_2 & -2.45 & $5.95 \mathrm{E}-44$ & & & \\
\hline TRINITY_DN21281_c0_g4_i5_2 & 2.28 & $3.62 \mathrm{E}-32$ & & & \\
\hline
\end{tabular}

Table 6: DEGs related to $\mathrm{K}^{+}$and $\mathrm{Na}^{+}$ion transport

\begin{tabular}{|c|c|c|c|c|c|}
\hline Gene ID & $\log _{2} \mathrm{FC}$ & P-value & Gene ID & $\log _{2} \mathrm{FC}$ & P-value \\
\hline KEA & & & $\mathrm{KOC}$ & & \\
\hline TRINITY_DN14177_c0_g1_i4_2 & -1.65 & $9.15 \mathrm{E}-22$ & TRINITY_DN14314_c0_g1_i7_1 & 1.52 & $1.39 \mathrm{E}-21$ \\
\hline TRINITY_DN14664_c0_g1_i3_2 & 1.82 & $2.80 \mathrm{E}-07$ & TRINITY_DN16211_c0_g1_i2_2 & 2.52 & $5.34 \mathrm{E}-06$ \\
\hline TRINITY_DN15615_c0_g1_i2_2 & -1.10 & $2.96 \mathrm{E}-04$ & TRINITY_DN16911_c0_g2_i5_2 & 7.38 & $1.02 \mathrm{E}-203$ \\
\hline TRINITY_DN17765_c1_g1_i9_1 & 3.65 & $5.91 \mathrm{E}-08$ & TRINITY_DN19502_c0_g3_i1_2 & 1.21 & $5.76 \mathrm{E}-06$ \\
\hline TRINITY_DN17765_c1_g2_i5_1 & -1.06 & $3.66 \mathrm{E}-02$ & TRINITY_DN20260_c0_g1_i20_2 & 2.08 & $3.86 \mathrm{E}-32$ \\
\hline TRINITY_DN19255_c0_g1_i3_1 & -3.11 & $3.36 \mathrm{E}-06$ & TRINITY_DN20505_c0_g1_i1_-1 & 3.24 & $4.61 \mathrm{E}-54$ \\
\hline TRINITY_DN19255_c0_g2_i4_1 & -3.14 & $7.72 \mathrm{E}-05$ & TRINITY_DN20585_c1_g2_i17_2 & 1.17 & $2.61 \mathrm{E}-05$ \\
\hline TRINITY_DN21559_c1_g5_i3_2 & 1.85 & $1.67 \mathrm{E}-05$ & TRINITY_DN22190_c2_g2_i1_2 & 3.45 & $3.48 \mathrm{E}-15$ \\
\hline TRINITY_DN22016_c0_g1_i24_2 & 3.69 & $2.89 \mathrm{E}-12$ & CNGC & & \\
\hline TRINITY_DN22016_c0_g2_i3_2 & 1.12 & $1.64 \mathrm{E}-04$ & TRINITY_DN12577_c0_g1_i1_2 & 2.10 & $6.61 \mathrm{E}-23$ \\
\hline KUP & & & TRINITY_DN19156_c0_g1_i14_2 & 1.99 & $1.11 \mathrm{E}-05$ \\
\hline TRINITY_DN15916_c0_g1_i8_1 & 1.80 & $5.39 \mathrm{E}-04$ & TRINITY_DN19236_c0_g1_i7_2 & 2.09 & $1.67 \mathrm{E}-37$ \\
\hline TRINITY_DN16437_c0_g1_i1_2 & 1.74 & $1.27 \mathrm{E}-07$ & TRINITY_DN20366_c0_g3_i7_1 & -1.15 & $1.52 \mathrm{E}-04$ \\
\hline TRINITY_DN16474_c0_g2_i1_2 & 2.08 & $6.62 \mathrm{E}-05$ & TRINITY_DN20693_c0_g1_i17_1 & -2.42 & $3.16 \mathrm{E}-08$ \\
\hline TRINITY_DN16474_c0_g3_i4_2 & 4.16 & $9.84 \mathrm{E}-103$ & TRINITY_DN21311_c0_g1_i4_2 & 1.53 & $7.62 \mathrm{E}-09$ \\
\hline TRINITY_DN16999_c0_g1_i4_1 & 2.05 & $8.74 \mathrm{E}-26$ & TRINITY_DN21317_c0_g2_i2_2 & 1.27 & $1.29 \mathrm{E}-07$ \\
\hline TRINITY_DN18621_c0_g1_i6_2 & 1.78 & 4.17E-09 & TRINITY_DN22290_c0_g1_i1_2 & 1.58 & $1.26 \mathrm{E}-06$ \\
\hline TRINITY_DN18799_c0_g1_i2_2 & -1.65 & $6.76 \mathrm{E}-07$ & P-ATPase & & \\
\hline TRINITY_DN19410_c0_g1_i7_1 & 1.17 & $1.81 \mathrm{E}-02$ & TRINITY_DN20505_c0_g2_i2_1 & 4.50 & $1.70 \mathrm{E}-135$ \\
\hline TRINITY_DN19574_c0_g1_i1_2 & 2.07 & 3.17E-14 & TRINITY_DN20585_c1_g2_i17_2 & 1.17 & $2.61 \mathrm{E}-05$ \\
\hline TRINITY_DN19850_c0_g1_i9_2 & 2.38 & $3.45 \mathrm{E}-23$ & TRINITY_DN22190_c2_g1_i9_2 & 3.48 & $6.03 \mathrm{E}-49$ \\
\hline TRINITY_DN19858_c0_g1_i37_2 & 2.97 & $4.48 \mathrm{E}-65$ & V-ATPase & & \\
\hline TRINITY_DN19995_c2_g1_i15_1 & -1.30 & $2.63 \mathrm{E}-06$ & TRINITY_DN13353_c0_g1_i2_2 & 5.91 & $2.81 \mathrm{E}-35$ \\
\hline TRINITY_DN21041_c0_g1_i3_2 & 1.94 & $5.25 \mathrm{E}-31$ & TRINITY_DN15633_c0_g1_i2_2 & 1.64 & $2.75 \mathrm{E}-09$ \\
\hline TRINITY_DN22105_c0_g1_i4_2 & 3.27 & $1.64 \mathrm{E}-54$ & TRINITY_DN14314_c0_g1_i7_1 & 1.52 & $1.39 \mathrm{E}-21$ \\
\hline TRINITY_DN24434_c0_g1_i1_1 & -2.95 & $1.47 \mathrm{E}-04$ & TRINITY_DN20260_c0_g1_i20_2 & 2.08 & $3.86 \mathrm{E}-32$ \\
\hline TRINITY_DN4618_c0_g1_ī1_1 & -3.05 & $6.68 \mathrm{E}-35$ & TRINITY_DN20505_c0_g1_i1_-̄ & 3.24 & $4.61 \mathrm{E}-54$ \\
\hline TRINITY_DN5960_c0_g1_i1_1 & 1.95 & 4.30E-20 & NHX & & \\
\hline TRINITY_DN7740_c0_g1_i1_1 & -3.82 & $6.23 \mathrm{E}-05$ & TRINITY_DN14878_c0_g1_i1_2 & -1.02 & $1.16 \mathrm{E}-03$ \\
\hline $\begin{array}{l}\text { TRINITY_DN7928_c0_g1_i2_1 } \\
\text { AKT }\end{array}$ & -2.28 & $1.36 \mathrm{E}-10$ & TRINITY_DN9153_c0_g1_i2_1 & -2.51 & $2.12 \mathrm{E}-04$ \\
\hline TRINITY_DN13806_c0_g1_i1_1 & -2.04 & $3.70 \mathrm{E}-08$ & & & \\
\hline
\end{tabular}

the largest group, followed by NAC (470 DEGs), MYBrelated (402 DEGs) and ERF (356 DEGs) family. The LSD (5 DFGs), LFY (5 DEGs) and SAP (1 DEGs) family had the least corresponding unigenes.

\section{Validation of DEGs relative expression by qRT-PCR}

The qRT-PCR results showed the expression patterns of 10 selected DEGs in good agreement with the relative expression results at transcriptome level (Fig. 9). This indicated that transcriptome data of Z. macrostachya roots obtained in this experiment were accurate.

\section{Discussion}

In this study, the roots informative transcriptome dataset for Z. macrostachya after $\mathrm{NaCl}$ treatment revealed that $68.76 \%$ of the 4,7325 unigenes were annotated by BLAST analysis, 
Table 7: DEGs related to osmotic adjustment substances

\begin{tabular}{lll}
\hline Gene ID & Log FC & P-value \\
\hline Free proline & & \\
TRINITY_DN12426_c0_g2_i1_1 & -2.54 & $2.81 \mathrm{E}-02$ \\
TRINITY_DN16249_c0_g1_i5_2 & 5.09 & $7.53 \mathrm{E}-51$ \\
TRINITY_DN16737_c0_g1_i1_2 & 4.69 & $3.33 \mathrm{E}-17$ \\
TRINITY_DN20583_c0_g1_i5_1 & 6.72 & $7.84 \mathrm{E}-272$ \\
TRINITY_DN22133_c0_g1_i14_2 & 3.78 & $3.74 \mathrm{E}-60$ \\
TRINITY_DN22200_c0_g1_i19_2 & 7.87 & $0.00 \mathrm{E}+00$ \\
TRINITY_DN20075_c0_g1_i5_1 & 2.19 & $8.55 \mathrm{E}-36$ \\
TRINITY_DN20857_c1_g4_i6_1 & 2.40 & $1.83 \mathrm{E}-47$ \\
TRINITY_DN19370_c0_g1_i3_1 & -2.85 & $1.21 \mathrm{E}-08$ \\
TRINITY_DN20069_c0_g1_i5_2 & 1.74 & $8.26 \mathrm{E}-22$ \\
TRINITY_DN15955_c0_g1_i5_1 & -1.31 & $5.68 \mathrm{E}-03$ \\
TRINITY_DN19808_c0_g2_i7_1 & -2.45 & $7.36 \mathrm{E}-10$ \\
Glycine betaine & & \\
TRINITY_DN12892_c0_g1_i2_1 & 2.29 & $1.74 \mathrm{E}-22$ \\
TRINITY_DN15030_c0_g1_i1_1 & -2.86 & $5.60 \mathrm{E}-05$ \\
TRINITY_DN18859_c0_g1_i5_2 & 1.82 & $9.97 \mathrm{E}-04$ \\
TRINITY_DN12184_c0_g1_i3_2 & 2.14 & $9.46 \mathrm{E}-19$ \\
\hline
\end{tabular}

Fig. 8: Distribution of TFs family

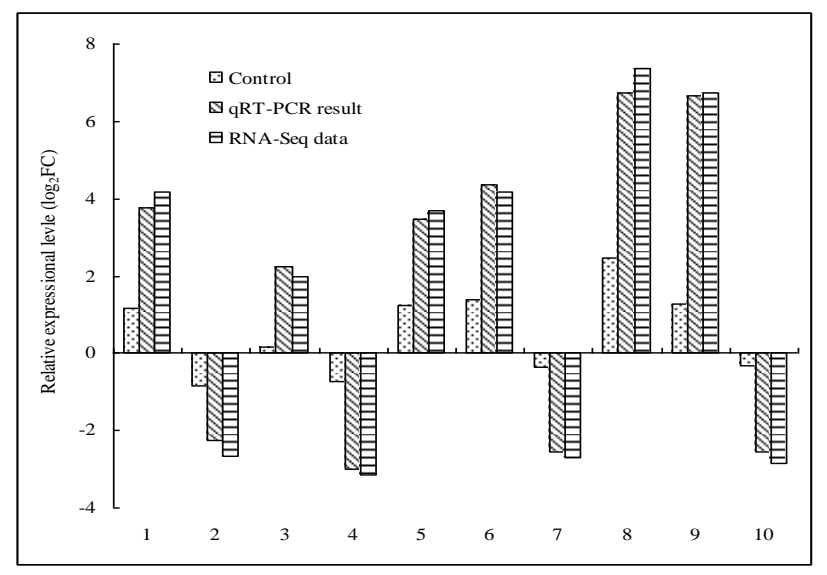

Fig. 9: Expression pattern validation of selected genes by qRTPCR

which suggested that the sequences of the Z. macrostachya unigenes generated in the present study were assembled and annotated correctly. A total of 14,558 DEGs were identified from $Z$. macrostachya roots under salt stress, these unigenes provided a comprehensive understanding towards the genes transcription profiles of Z. macrostachya, and laid a solid foundation for further study of salt-tolerance mechanisms and identification of new genes in this species.

ROS accumulation induce cytoplasmic membrane damage (Xiong et al. 2020). Study has shown that salttolerance of plant was related to scavenging capacity of antioxidative proteins to some extent (Zhang et al. 2012; Bose et al. 2014). SOD is the first-line of defense in resistance to oxidative injury, which can dismutate $\cdot \mathrm{O}_{2}{ }^{-}$into $\mathrm{O}_{2}$ and $\mathrm{H}_{2} \mathrm{O}_{2}(\mathrm{Qu}$ et al. 2010), CAT and POD can dismutate $\mathrm{H}_{2} \mathrm{O}_{2}$ into $\mathrm{H}_{2} \mathrm{O}$ and $\mathrm{O}_{2}$ (Xu et al. 2013), whereas, APX exerts a vital part in the catalysis of $\mathrm{H}_{2} \mathrm{O}_{2}$ conversion to $\mathrm{H}_{2} \mathrm{O}$, and $\mathrm{H}_{2} \mathrm{O}_{2}$ can also be reduced by GPX in the GPX pathway (Gu et al. 2018). In this study, these DEGs encodesd enzymatic antioxidants might be the vital factors involved in ROS elimination in Z. macrostachya.

Ions transport is a crucial factor in plant response to salt stress. To deal with salt stress, plants have evolved specific mechanisms for coordinating the $\mathrm{Na}^{+}$discharge and $\mathrm{K}^{+}$uptake processes (Guo et al. 2016; Lv et al. 2018). KUP, KEA and AKT constitute the $\mathrm{K}^{+}$uptake system in plant, PM- $\mathrm{H}^{+}$-ATPase and $\mathrm{V}-\mathrm{H}^{+}$-ATPase can generate the driving force to extrude $\mathrm{Na}^{+}$out of cell and compartmentalize $\mathrm{Na}^{+}$ to vacuole, and these are regarded as the mechanisms that a plant employs to restore the homeostasis of ions in cell (Chinnusamy et al. 2006). In our study, 37 up-regulated DEGs involved in $\mathrm{K}^{+}$uptake, 7 up-regulated DEGs involved in $\mathrm{Na}^{+}$extrusion was identified, respectively, indicating these DEGs played critical roles in reestablishing the cellular $\mathrm{K}^{+}$and $\mathrm{Na}^{+}$homeostasis. In addition, NHX were probably responsible for $\mathrm{Na}^{+}$sequestration, but 2 DEGs encoding NHX were down-regulated, revealing that NHXs were not likely involved in $\mathrm{Na}^{+}$discharge.

It is well-known that plants synthesize some osmolytes, such as free proline and Glycine betaine, so as to alleviate the negative effects of toxic ions and osmotic imbalance (Ingram and Bartels 1996; Ashraf and Foolad 2007). In this study, 11 DEGs involved in free proline and Glycine betaine metabolism were identified, their expression were up-regulated, it suggested that these DEGs exerted vital parts in regulating osmotic balance in $\mathrm{Z}$. macrostachya.

The TFs can modulate the downstream genes expression, which play important roles in plant response to abiotic stresses (Capella et al. 2015). Specifically, some TFs families have been identified from halophytes through RNA-Seq, such as Iris lactea var. chinensis (Gu et al. 2018), Reaumuria trigyna (Wang et al. 2014) and Suaeda fruticosa (Diray-Arce et al. 2015). In our study, some DEGs were annotated to the BHLH, NAC, MYB-relate or ERF TFs family. It is reported that the expression of TabHLH13 isolated from wheat was rapidly up-regulated after salt stress (Kim and Kim 2006), while that of bHLH92 in Arabidopsis thaliana was triggered under drought and high salinity (Jiang and Deyholos 2006; Liu et al. 2014), indicating that the BHLH TFs family exert vital parts in the salt-tolerance 
of plant. Previous studies showed that, the over-expression of NAC TF GhATAF1 improved the salt-tolerance of cotton (He et al. 2016); besides, the ERFs TFs family can modulate the responses to abiotic stress and ethylene, as well as disease resistance, which were achieved through targeting the downstream promoter GCC-box (Ohme-Takagi and Shinshi 1995; Stockinger et al. 1997). By contrast, the expression of MYB-related TF AtMYBL in A. thaliana is activated by salt stress (Zhang et al. 2011). Thus, the TFs identified in our study laid the basis for more intensive studies.

\section{Conclusion}

A total of 4,7325 unigenes were assembled, and $68.76 \%$ of the 4,7325 unigenes were annotated by BLAST analysis. Altogether 14,558 DEGs were identified between salt stress and control samples, many DEGs were identified to encode enzymes related to the ROS scavenging system, $\mathrm{K}^{+}$and $\mathrm{Na}^{+}$ transport proteins, osmotic adjustment substances and TFs, which potentially played vital roles in salt stress response in $Z$. macrostachya. Our findings provide useful insights for salt-tolerance molecular mechanisms of $Z$. macrostachya and provide basis for future cloning of salt-tolerance genes in this species.

\section{Acknowledgements}

This work was funded by the Jiangsu Key Laboratory for Bioresources of Saline Soils Fund (JKLBS2017009), and the Six Talent Peaks Project in Jiangsu province (NY-096) in 2017.

\section{Author Contributions}

HG $\mathrm{Hu}$ and ZM Zhang designed the experiments; HG $\mathrm{Hu}$, ZM Zhang and SN Min performed the experiments; $\mathrm{HG} \mathrm{Hu}$ and ZM Zhang analyzed RNA-Seq data; HG Hu, ZM Zhang and SN Min wrote the manuscript.

\section{References}

Altschul SF, W Gish, W Miller, EW Myers, DJ Lipman (1990). Basic local alignment search tool. J Mol Biol 215:403-410

Anders S, W Huber (2012). Differential expression of RNA-Seq data at the gene level-the DESeq package. EMBL 3:1-23

Ashraf M, MR Foolad (2007). Roles of glycine bataine and proline in improving plant abiotic stress resistance. Environ Exp Bot 59:206-216

Bolger AM, M Lohse, B Usadel (2014). Trimmomatic: A flexible trimmer for Illumina sequence data. Bioinformatics 30:2114-2120

Bose J, A Rodrigo-Moreno, S Shabala (2014). ROS homeostasis in halophytes in the context of salinity stress tolerance. $J$ Exp Bot 65:1241-1257

Capella M, PA Ribone, AL Arce, RL Chan (2015). Arabidopsis thaliana HomeoBox 1(AtHB1), a Homedomain-Leucine Zipper I (HD-Zip I) transcription factor, is regulated by PHYTOCHROMEINTERACTING FACTOR 1 to promote hypocotyl elongation. New Phytol 207:669-682
Chinnusamy V, JH Zhu, JK Zhu (2006). Salt stress signaling and mechanisms of plant salt tolerance. Genet Eng 27:141-177

Deinlein U, AB Stephan, T Horie, W Luo, G Xu, JI Schroeder (2014). Plant salt-tolerance mechanisms. Trends Plant Sci 19:371-379

Diray-Arce J, M Clement, B Gul, MA Khan, BL Nielsen (2015). Transcriptome assembly, profiling and differential gene expression analysis of the halophyte Suaeda fruticosa provides insights into salt tolerance. BMC Genomics 16; Article 353

Flowers TJ, TD Colmer (2008). Salinity tolerance in halophytes. New Phytol 179:945-963

Grabherr MG, BJ Haas, M Yassour, JZ Levin (2011). Trinity: Reconstructing a full-length transcriptome without a genome from RNA-Seq data. Nat Biotechnol 29:644-652

Gu C, S Xu, Z Wang, L Liu, Y Zhang, Y Deng, S Huang (2018). De novo sequencing, assembly, and analysis of Iris lactea var. chinensis roots' transcriptome in response to salt stress. Plant Physiol Biochem 125:1-12

Guo HL, Y Wang, DD Li, JB Chen, JQ Zong, ZY Wang, X Chen, JX Liu (2016). Growth response and ion regulation of seashore paspalum accessions to increasing salinity. Environ Exp Bot $131: 137-145$

He X, L Zhu, L Xu, W Guo, X Zhang (2016). GhATAF1, a NAC transcription factor, confers abiotic and biotic stress responses by regulating phytohormonal signaling Networks. Plant Cell Rep $35: 2167-2179$

Hu HG, ZM Zhang (2009). Study on the physiologicai adaptability of Zoysia macrostachya to salt stress. J Anhui Agric Sci 37:6413-6415

Hu HG, ZM Zhang (2010). Study on responses to salt stress and critical salt concentration of Zoysia macrostachya. Northern Hortic 3:80-83

Hu HG, ZM Zhang, TX Sun, YY Han (2016). Effects of mixed saline stress on the absorption and transportation of $\mathrm{K}^{+}$and $\mathrm{Na}^{+}$of Zoysia Willd. Chin J Trop Crops 37:2145-2149

Ingram J, D Bartels (1996). The molecular basis of dehydration tolerance in plants. Annu Rev Plant Biol 7:377-403

Jiang Y, MK Deyholos (2006). Comprehensive transcriptional profiling of $\mathrm{NaCl}$-stressed Arabidopsis roots reveals novel classes of responsive genes. BMC Plant Biol 6:25-30

Jin J, H Zhang, L Kong, G Gao, J Luo (2014). PlantTFDB 3.0: A portal for the functional and evolutionary study of plant transcription factors. Nucl Acids Res 42:1182-1187

Kanehisa M, M Araki, S Goto, M Hattori (2008). KEGG for linking genomes to life and the environment. Nucl Acids Res 36:480-484

Kim J, HY Kim (2006). Functional analysis of a calcium-binding transcription factor involved in plant salt stress signaling. FEBS Lett 580:5251-5256

Langmead B, SL Salzberg (2012). Fast gapped-read alignment with Bowtie 2. Nat Meth 9:357-359

Liu W, H Tai, S Li, W Gao, M Zhao, CX Xie, W Li (2014). bHLH122 is important for drought and osmotic stress resistance in Arabidopsis and in the repression of ABA catabolism. New Phytol 201:1192-1204

Liu ZX, YJ Hua, SN Wang, XH Liu, LS Zou, CH Chen, H Zhao, Y Yan (2020). Analysis of the Prunellae spica transcriptome under salt stress. Plant Physiol Biochem 156:314-322

Livak KJ, TD Schmittgen (2001). Analysis of relative gene expression data using real-time quantitative PCR and the $2^{-\Delta \Delta C T}$ method. Methods 25:402-408

Lv XY, Y Jin, YG Wang (2018). De novo transcriptome assembly and identification of salt-responsive genes in sugar beet M14. Comput Biol Chem 75:1-10

Meera SP, A Augustine (2020). De novo transcriptome analysis of Rhizophora mucronata Lam. Furnishes evidence for the existence of glyoxalase system correlated to glutathione metabolic enzymes and glutathione regulated transporter in salt tolerant mangroves. Plant Physiol Biochem 155:683-696

Miller G, V Shulaev, R Mittler (2008). Reactive oxygen signaling and abiotic stress. Physiol Plantarum 133:481-489

Ohme-Takagi M, H Shinshi (1995). Ethylene-inducible DNA binding proteins that interact with an ethylene-responsive element. Plant Cell $7: 173-182$ 
Qu CP, ZR Xu, GJ Liu, C Liu, Y Li, ZG Wei, GF Liu (2010). Differential expression of copper-zinc superoxide dismutase gene of Polygonum sibiricum leaves, stems and underground stems, subjected to highsalt stress. Intl J Mol Sci 11:5234-5245

Roberts A, P Lior (2013). Streaming fragment assignment for real-time analysis of sequencing experiments. Nat Meth 10:71-73

Stockinger EJ, SJ Gilmour, MF Thomashow (1997). Arabidopsis thaliana CBF1 encodes an AP2 domain-containing transcriptional activator that binds to the C-repeat/DRE, a cis-acting DNA regulatory element that stimulates transcription in response to low temperature and water deficit. Proc Natl Acad Sci USA 94:1035-1040

Tang XL, XM Mu, HB Shao, HY Wang, M Brestic (2015). Global plantrespondingmechanisms to salt stress: Physiological and molecular levels and implications in biotechnology. Crit Rev Biotechnol 35:425-437

Trapnell C, BA Williams, G Pertea, A Mortazavi, G Kwan, MJ van Baren, SL Salzberg, BJ Wold, L Pachter (2010). Transcript assembly and quantification by RNA-Seq reveals unannotated transcripts and isoform switching during cell differentiation. Nat Biotechnol 28:511-515

Wang J, LL Zheng, TP Gu, XF Wang, YC Wang (2014). Cloning and expression analysis of two WRKY transcription factors from the rare recretohalophyte Reaumuria trigyna. Acta Pratacult Sin 4:122-129
Xiong X, YQ Wei, JH Chen, N Liu, YJ Zhang (2020). Transcriptome analysis of genes and pathways associated with salt tolerance in alfalfa under non-uniform salt stress. Plant Physiol Biochem $151: 323-333$

Xu R, M Yamada, H Fujiyama (2013). Lipid peroxidation and antioxidative enzymes of two turfgrass species under salinity stress. Pedosphere 23:213-222

Zhang F, JL Ding, T Tashpolat, BC Wang (2007). Analysis on characteristics of soil salinization in the arid regions: A case study in the delta oasis of Weigan and Kuqa rivers. Acta Pratacult Sin 16:34-40

Zhang H, B Han, T Wang, SX Chen, HY Li, YH Zhang, SJ Dai (2012). Mechanisms of plant salt response: Insights from proteomics. $J$ Proteome Res 11:49-67

Zhang X, HW Ju, MS Chung, P Huang, SJ Ahn, CS Kim (2011). The R-Rtype MYB-like transcription factor, AtMYBL, is involved in promoting leaf senescence and modulates an abiotic stress response in Arabidopsis. Plant Cell Physiol 52:138-148

Zhu JK (2001). Plant salt tolerance. Trends Plant Sci 6:66-71

Zhu JK (2003). Regulation of ion homeostasis under salt stress. Curr Opin Plant Biol 6:441-445 